\title{
Mild exacerbation of obesity- and age-dependent liver disease progression by senolytic cocktail dasatinib + quercetin
}

\author{
Marco Raffaele ${ }^{1 *+} \oplus$, Kristina Kovacovicova ${ }^{1 \dagger}$, Jan Frohlich 1 , Oriana Lo Re ${ }^{1}$, Sebastiano Giallongo 1,2, \\ Jude A. Oben ${ }^{3}$, Martin Faldyna ${ }^{4}$, Lenka Leva ${ }^{4}$, Antonino Giulio Giannone ${ }^{5}$, Daniela Cabibi ${ }^{5}$ and \\ Manlio Vinciguerra ${ }^{1,3,6^{*}}$
}

\begin{abstract}
Background: Nonalcoholic fatty liver disease (NAFLD) is increasingly prevalent and represents a growing challenge in terms of prevention and treatment. A minority of affected patients develops inflammation, subsequently fibrosis, cirrhosis and hepatocellular carcinoma (HCC). HCC is a leading cause of cancer-related death. An increased number of senescent cells correlate with age-related tissue degeneration during NAFLD-induced HCC. Senolytics are promising agents that target selectively senescent cells. Previous studies showed that whereas a combination of the senolytic drugs dasatinib and quercetin $(D+Q)$ reduced NAFLD in mice, $D+Q$ lacked efficacy in removing doxorubicininduced $\beta$-gal-positive senescent cells in human HCC xenografted mice. Whether $D+Q$ has an effect on the ageassociated spectrum of NAFLD-inflammation-HCC remains unknown.

Methods: Here, we utilized an established model of age- and obesity-associated HCC, the low dose diethylnitrosamine (DEN)/high fat diet (HFD), a regimen promoting liver inflammation and tumorigenesis over a long period of 9 months. Four groups of mice each were created: group 1 included control untreated mice; group 2 included mice treated with D+Q; group 3 included mice undergoing the DEN/HFD protocol; group 4 included mice undergoing the DEN/HFD protocol with the administration of D+Q. At the end of the chemical/dietary regimen, we analyzed liver damage and cell senescence by histopathology, QPCR and immunoblotting approaches.
\end{abstract}

Results: Unexpectedly, D+Q worsened liver disease progression in the DEN/HFD mouse model, slightly increasing histological damage and tumorigenesis, while having no effect on senescent cells removal.

Conclusions: In summary, using an animal model that fully recapitulates NAFLD, we demonstrate that these compounds are ineffective against age-associated NAFLD-induced HCC.

Keywords: Senolytics, Liver diseases, Inflammation, Cancer, Obesity

*Correspondence: marco.raffaele@fnusa.cz; manlio.vinciguerra@fnusa.cz ${ }^{\dagger}$ Marco Raffaele and Kristina Kovacovicova have contributed equally to this work.

${ }^{1}$ International Clinical Research Center, St. Anne's University Hospital, Brno, Czech Republic

Full list of author information is available at the end of the article

\section{Background}

The majority chronic diseases appear with increasing age and thus have high prevalence in the elderly $[1,2]$. Similarly, geriatric conditions, such as mild cognitive impairment and frailty drastically augment with the aging of the individual [3]. Non-alcoholic fatty liver disease (NAFLD) is the most common chronic liver disorder, and it affects $>25 \%$ of the general population [4]. NAFLD is the main risk factor original author(s) and the source, provide a link to the Creative Commons licence, and indicate if changes were made. The images or other third party material in this article are included in the article's Creative Commons licence, unless indicated otherwise in a credit line to the material. If material is not included in the article's Creative Commons licence and your intended use is not permitted by statutory regulation or exceeds the permitted use, you will need to obtain permission directly from the copyright holder. To view a copy of this licence, visit http://creativecommons.org/licenses/by/4.0/. The Creative Commons Public Domain Dedication waiver (http://creativeco mmons.org/publicdomain/zero/1.0/) applies to the data made available in this article, unless otherwise stated in a credit line to the data. 
to develop fibrosis, cirrhosis and hepatocellular carcinoma (HCC), a devastating disease [5]. The incidence of NAFLD increases with age [5]. A great bulk of evidence shows that the progressive accumulation of senescent cells can mark and drive age-associated alterations and pathologies [6-8]. Senescence is a cellular response characterized by a stable growth arrest and other phenotypic alterations that include upregulation of tumor suppressor p16 and a proinflammatory secretome (senescence-associated secretory phenotype, SASP) [9]. Senescence plays roles in normal development, maintains tissue homeostasis, and limits tumor progression. Rapid gain of interest in cellular senescence is rising from the potential of therapeutically targeting this process, in order to improve age-related pathologies, using drugs called senolytics [10-13]. These agents include dasatinib (D), quercetin (Q), fisetin, Navitoclax (ABT-263) and others [10-12]. Senescence associated $\beta$-galactosidase (SA- $\beta$-gal) - the most common marker of cellular senescence [14] - assays in human or murine fibroblasts are increasingly used as high-throughput screening platforms to rapidly identify new senolytics $[15,16]$, for further testing. Nonetheless, the first and best-studied senolytic drugs, D and Q, were discovered using a mechanism-based approach instead a high-throughput screening [17]. It was shown that cellular senescence drives NAFLD and the mix Dasatinib $(5 \mathrm{mg} / \mathrm{kg})+$ Quercetin $(50 \mathrm{mg} /$ $\mathrm{kg}$ ) dependent elimination of p16 overexpressing senescent cells may be a novel therapeutic strategy to reduce NAFLD and obesity-induced anxiety in mice models. The same authors reported that "hit-and-run" treatment with $\mathrm{D}+\mathrm{Q}$, which have elimination half-lives $<11 \mathrm{~h}$, significantly decreases senescent cell burden in humans, in particular in p16 highly expressing cells (p16 ${ }^{\text {high }}$ ) in the adipose tissue. On the contrary, we demonstrated that $\mathrm{D}+\mathrm{Q}$ used at the same concentration of these previous studies [17-21], are ineffective, alone or in synergy with senescence-inducing chemotherapy, in mice xenografted with human HCC cells [22]. However, HCC xenografts present several approximations, including immuno-deficient hosts, non-natural tumor site (sub-cutaneous) and lack of genetic diversity. Moreover, they do not feature the progression of liver diseases. The current study was designed to assess the role of $\mathrm{D}+\mathrm{Q}$ in a comprehensive and robust mouse model of obesity- and age- dependent liver disease progression, developing NAFLD and HCC [23, 24].

\section{Results}

Dasatinib + Quercetin $(D+Q)$ worsens liver disease progression in the diethylnitrosamine (DEN) / high fat diet (HFD) mouse model

Cellular senescence was shown to drive NAFLD and $\mathrm{D}+\mathrm{Q}$ treatment could alleviate this pathology [18-20] . However, these previous reports did not analyze the effectiveness of these senolytics in hampering the progression of NAFLD into inflammatory states and, ultimately, into HCC. To test this hypothesis, we adopted a well-established mouse model of NAFLD-induced HCC, where young mice ( 7 weeks of age) are administered with a low dose of a carcinogen $(25 \mathrm{mg} / \mathrm{kg}$ diethynitrosamine, DEN), followed by feeding a high fat diet (HFD, 60\% energy from lard)) from the 7th week to the 43rd week, when the animals were sacrificed for further analyses [23, 24] (Fig. 1a). Four groups of 20 mice each were created: group 1 included control untreated mice; group 2 included mice treated with $\mathrm{D}+\mathrm{Q}$ from the 24th week to the $43 \mathrm{rd}$ week of age $(\mathrm{D}=5 \mathrm{mg} / \mathrm{kg} ; \mathrm{Q}=50 \mathrm{mg} / \mathrm{kg})$; group 3 included mice undergoing the above mentioned DEN/ HFD protocol; group 4 included mice undergoing the DEN/HFD protocol with the administration of $D+Q$ (Fig. 1a). At the end of the chemical/dietary regimen, the DEN/HFD groups 3 and 4 exhibited a $\sim 50 \%$ increase in body weight, with not tangible effect of $\mathrm{D}+\mathrm{Q}$ treatment (Fig. 1b). Blood analysis showed no differences in number of white blood cell and red blood cell count as well as in hemoglobin levels, among the four groups (Table 1). Upon HFD feeding, biochemical plasma analyses detected a significant increase in the levels of LDL, HDL, TC, ALT and AST, while we observed a paradoxical decrease in TG - most likely due to a reduced dietary carbohydrate [25], compared to control diet (Table 2). None of these changes were affected by $D+Q$ treatment (Table 2). We then investigated the ability of the four groups of mice to respond to a glucose challenge, as readout of metabolic health. In glucose tolerance tests (GTT), glucose levels were significantly higher in DEN/ HFD mice (group 3) at 90 and 120 min time points, compared to control littermates (group 1); notably, D+Q administration (in groups 2 and 4) did not have any significant effect on GTT curves (Fig. 1c). We then conducted histopathological assessment of D+Q effects on liver parenchymal architecture. Figure 2a shows representative images of Hematoxylin \& Eosin (H\&E) stained liver sections of mice fed with control diet or HFD and treated with $\mathrm{D}+\mathrm{Q}$. Steatosis reached the maximum score of 3 in the HFD, while a value of 1 was assigned to the control diet groups identifying a mild steatosis probably caused by aging. Several hepatocyte ballooning was also noticed in the livers of HFD mice, while only a small number was detected in liver control-diet mice livers. In both diet groups $\mathrm{D}+\mathrm{Q}$ did not show to improve liver health, obtaining similar steatosis and ballooning scores compared to their respective controls. The increased fat storage in the liver of mice fed with HFD was corroborated by the increased expression levels of key lipogenic genes Ppar- $\gamma$ and Cebpa, the lipid droplets growth related genes Plin2, Ceidec and the main membrane fatty 


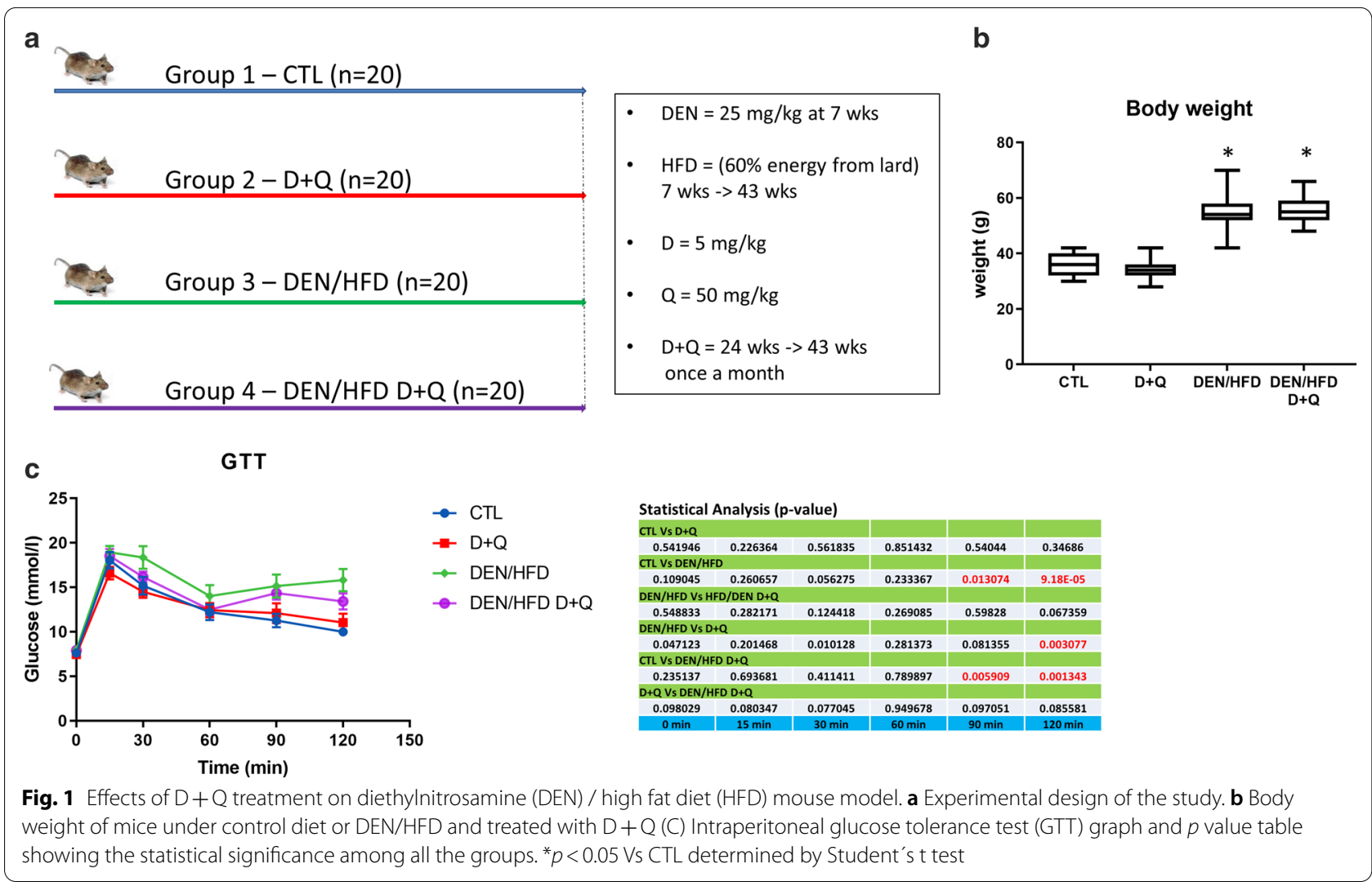

acid transporter Cd36; while Pgc-1 $\alpha$, a master regulator of the mitochondrial functions was downregulated in the groups upon HFD feeding, compared to control (Fig. 3) [26]. In accordance with the fibrotic processes that characterized the progression of NADLF, the levels of the markers Col1a1 and Timp1 were strongly upregulated in the HFD groups compared to control [27]. None of these changes were affected by $\mathrm{D}+\mathrm{Q}$ treatment (Fig. 3). Dysplastic nodules were observed in many mice liver and classified based on their size, in small nodules $(<3 \mathrm{~mm})$ and large nodules (>3 mm) (Fig. $2 \mathrm{~b}$ ). About $35 \%$ of HFD mice livers pre-treated with DEN and, surprisingly, $5 \%$ of lean mice treated with $\mathrm{D}+\mathrm{Q}$, were presenting nodules, while none was detected in the lean controls. Interestingly, whereas the percentage of tumor-bearing mice in HFD/DEN control and treated group was similar, a difference in small and large nodules ratio was noted, with an increased presence of large nodules in the HFD/DEN $\mathrm{D}+\mathrm{Q}$ group. The senolytic effect of $\mathrm{D}+\mathrm{Q}$ treatment was assessed by SA- $\beta$-gal staining (Fig. $2 \mathrm{c}$ ), and by gene/protein expression measurement of the senescence marker p16 (Fig. 2d). Contrary to previous studies [18], the analysis of B-gal staining images did not reveal any significant difference in senescence positive cells between the treated mice and their control, as shown in the representative images in Fig. 2c. Supporting the SA- $\beta$-gal staining, the evaluation of p16 gene and protein levels did not show significant differences among the groups (Fig. 2d). These findings suggest that $\mathrm{D}+\mathrm{Q}$ is not effective in removing senescent cells from adult mice (11 months) liver, further showing a slight pro-tumorigenic activity in accordance with our previous finding [22].

\section{Methods}

\section{Mice models}

All experiments were carried out following the rules of reduction of numbers of animals and minimizing their suffering during the experiments.

$D+Q$ treatment: all animal work was conducted either according to Act No 246/1992 Coll., on the protection of animals against cruelty and was approved by the Central Commission for Animal Welfare, approval ID 2989/2018 (Ministry of Agriculture, Czech Republic). Male C57/ BL6J mice were obtained from AnLab (Czech Republic) at the age of 5 weeks and were maintained in a pathogenfree facility under temperature- and light- controlled conditions $\left(22+2{ }^{\circ} \mathrm{C}, 12 \mathrm{~h}\right.$ light/dark regimen) with free access to food and water. The animals were fed by control diet (CD.88137; ssniff Spezialdiäten $\mathrm{GmbH}$ ) or by experimental high-fat diet (60\% of kj has the fat origin; D12492; 


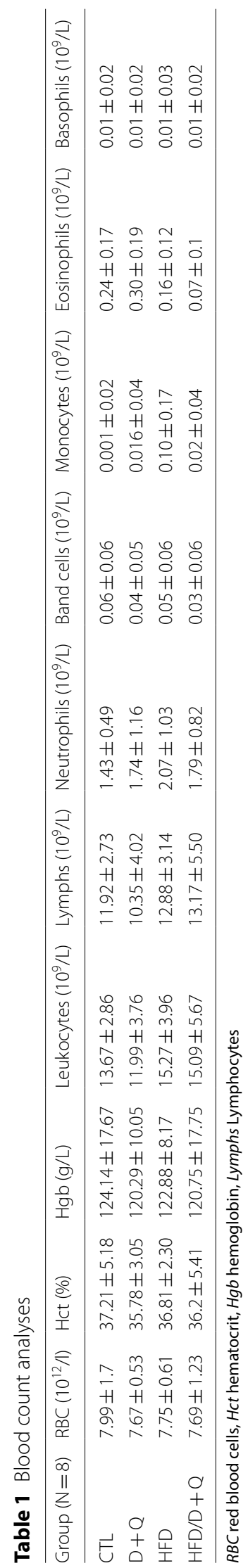


Table 2 Plasma analyses

\begin{tabular}{lllllll}
\hline Group $(\mathrm{N}=6)$ & $\mathrm{LDL}(\mathrm{mmol} / \mathrm{L})$ & $\mathrm{HDL}(\mathrm{mmol} / \mathrm{L})$ & $\mathrm{TC}(\mathrm{mmol} / \mathrm{L})$ & $\mathrm{TG}(\mathrm{mmol} / \mathrm{L})$ & ALT $(\mu \mathrm{kat} / \mathrm{L})$ & AST $(\mu \mathrm{kat} / \mathrm{L})$ \\
\hline CTL & $0.40 \pm 0.09$ & $2.4 \pm 0.41$ & $3.9 \pm 0.45$ & $1.16 \pm 0.24$ & $1.50 \pm 0.49$ & $0.44 \pm 0.12$ \\
D+Q & $0.37 \pm 0.06$ & $2.52 \pm 0.54$ & $3.64 \pm 0.42$ & $0.96 \pm 0.22$ & $1.72 \pm 0.96$ & $0.66 \pm 0.49$ \\
HFD & $0.63 \pm 0.07^{*}$ & $4.56 \pm 0.28^{*}$ & $6.70 \pm 0.47^{*}$ & $0.88 \pm 0.06^{*}$ & $3.09 \pm 0.99^{*}$ & $4.33 \pm 1.56^{*}$ \\
HFD/D + Q & $0.65 \pm 0.07^{*}$ & $4.66 \pm 0.11^{*}$ & $6.98 \pm 0.19^{*}$ & $0.80 \pm 0.06^{*}$ & $2.98 \pm 0.59^{*}$ & $4.47 \pm 0.93^{*}$
\end{tabular}

$L D L$ low-density lipoprotein, HDL high-density lipoprotein, $T C$ total cholesterol, $T G$ triglycerides, $A L T$ alanine transaminase, $A S T$ aspartate transaminase * $p<0.05$ vs CTL Group
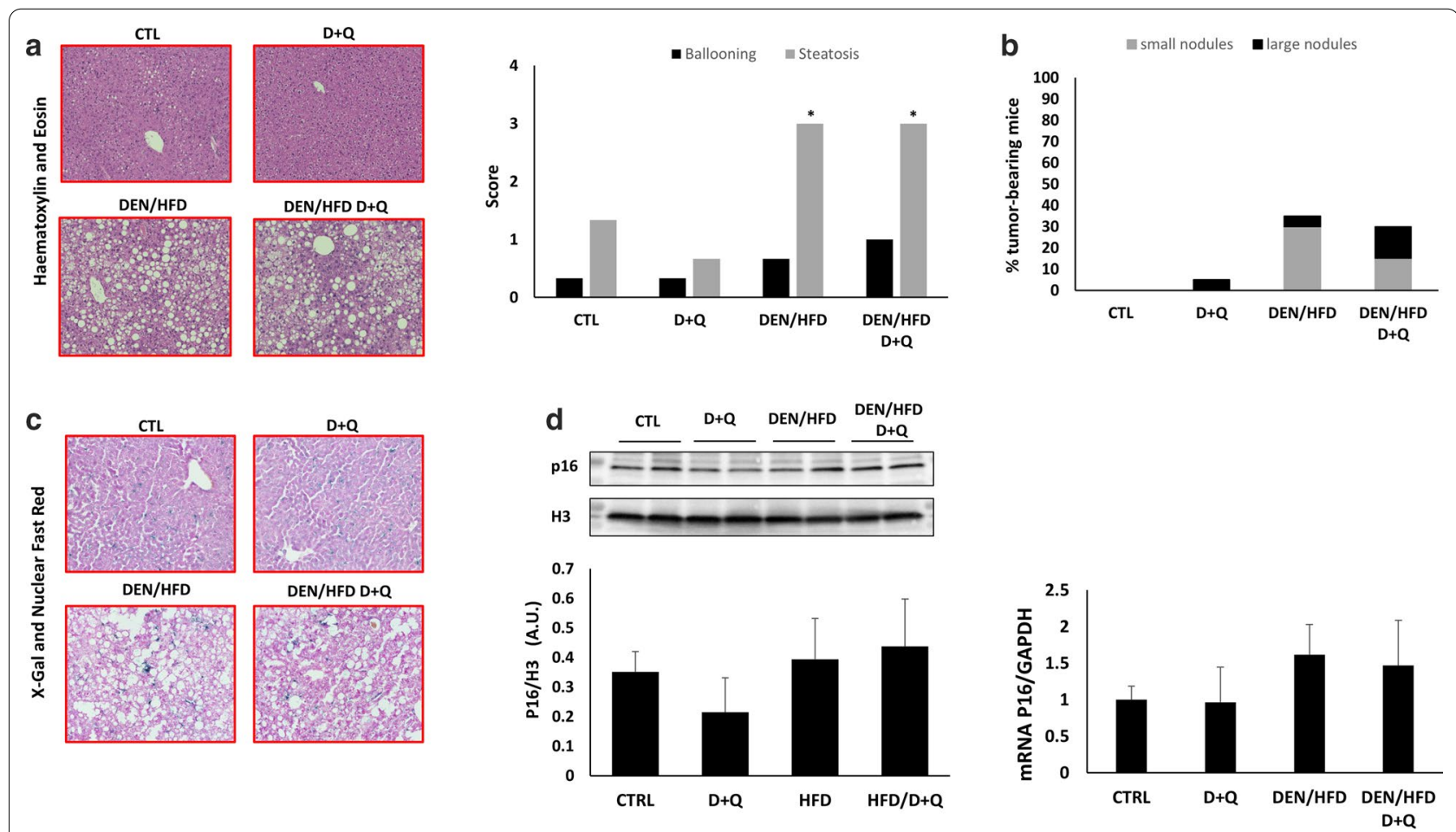

Fig. 2 Effects of $D+Q$ treatment worsen liver disease progression. a Representative liver H\&E images from control diet and DEN/HFD mice treated with $D+Q$ and steatosis/ballooning score graph. $\mathbf{b}$ Distribution of tumor-bearing mice among the groups, with classification of dysplastic nodules based on the size in small nodules (diameter $<3 \mathrm{~mm}$ ) and large nodules (diameter $>3 \mathrm{~mm}$ ). c Representative liver $\beta$-gal staining + nuclear fast red (NFR) images of CTL, D $+Q, D E N / H F D$ and HFD/DEN D $+Q$ groups showing no difference in senescent positive cells. $\mathbf{d}$ Immunoblotting and qRT-PCR analysis of the senescence marker p16. ${ }^{*} p<0.05 \mathrm{Vs} C T L$ determined by Student's t test

ssniff Spezialdiäten $\mathrm{GmbH})$. Mice were divided into following groups: (1) control group: mice given a placebo treatment; (2) D+Q group: mouse given dasatinib plus quercetin $(\mathrm{D}+\mathrm{Q})$ treatment; (3) DEN/HFD group: the initial liver damage was induced by single administration of DEN and animals were fed by high fat diet [23]; (4) DEN/HFD D $+Q$ group: the same liver damage induction as in group 3 plus $\mathrm{D}+\mathrm{Q}$ treatment. Mice from group 3 and 4 (model of steatosis and steatohepatitis) obtained single i.p. injection of diethylnitrosamine (DEN; $25 \mathrm{mg} /$ $\mathrm{kg}$; Sigma Aldrich) at the age of 7 weeks. The intervention of senolytics or placebo started at the 6 months of age and it was performed once per month over a 5-month period. The solution of dasatinib ( $5 \mathrm{mg} / \mathrm{kg}$; Sigma Aldrich) and quercetin (50 mg/kg; Sigma Aldrich) or the placebo was administrated by oral gavage in $100-150 \mu \mathrm{L}$. Glucose tolerance test (GTT) was performed one week before the sacrifice, after $12 \mathrm{~h}$ of fasting by i.p. administration of $1.5 \mathrm{~g} / \mathrm{kg}$ of glucose solution (Sigma Aldrich). Blood glucose levels were obtained at time points 0 (basal level); 15; 30; 45; 60 and 120 min measured by ACCUCHEK Performa glucometer (Roche). Blood samples were collected during the sacrifice and hematological and biochemical parameters were analyzed by hematological analyzer BC-2800 Vet and biochemical analyzer BS200 


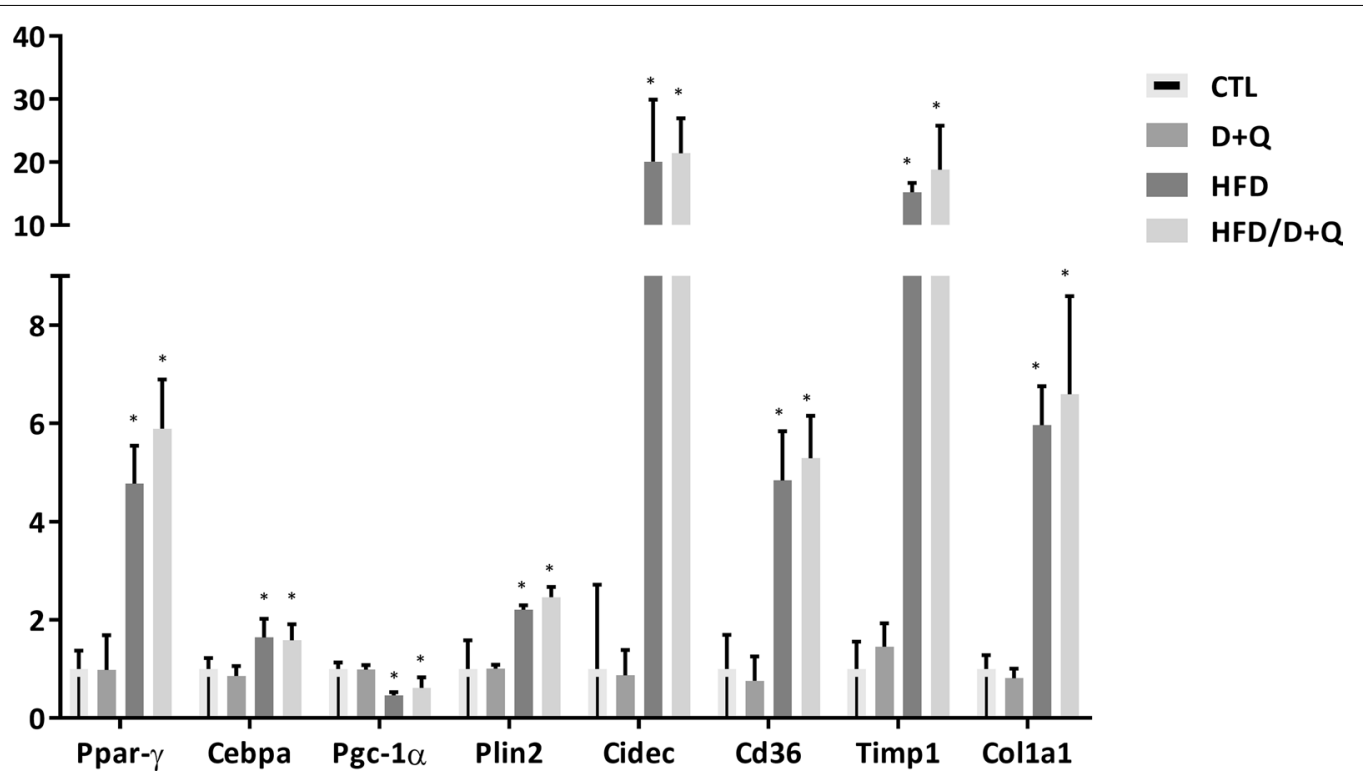

Fig. $3 \mathrm{D}+\mathrm{Q}$ does not affect NAFLD key factors gene expression in liver of both lean and obese mice. mRNA expression of Ppar- $y$, Cebpa, Pgc-1a, Plin2, Cidec, Cd36, Timp1 and Col1a1 of control mice, D + Q treated group, DEN/HFD group and DEN/HFD mice treated with $D+Q$. ${ }^{*} p<0.05$ Vs CTL determined by Student's $t$ test

(Mindray, Shenzhen, PRC), respectively, according to manufacturer's recommendations.

\section{Histology}

Samples of livers were harvested from euthanized mice, fixed with $4 \%$ paraformaldehyde, processed for the embedding in tissue freezing medium (Leica) or paraffin wax (Surgipath Paraplast Plus, Leica) and sectioned for histopathological analysis. The paraffine liver sections were stained with Hematoxylin-Eosin (H\&E; Sigma) for overall assessment of parenchymal architecture, hepatocyte abnormalities (including ballooning), inflammatory infiltration and the measurement of lipid droplets size. These features were scored according to the NAFLD histologic activity score (NAS) system [28]. Briefly, double-blinded analysis identified the score of steatosis (grade $0 \leq 5 \% ; 1=5-33 \%$; grade $2=34 \%-66 \%$; grade $3 \geq 66 \%$ ), lobular inflammation ( 0 : no foci, $1<2$ foci per $200 \times$ field, $2: 2$ to 4 foci per $200 \times$ field, and 3:>4 foci per $200 \times$ field), hepatocyte ballooning (0: none; 1 : rare or few; 2: many). Fibrosis was quantified using ImageJ software (NIH) and expressed as \% of the total area of the section. All analyses were performed in triplicate by two independent pathologists (A.G.G. and D.C.).

\section{X-gal staining}

B-galactosidase detection method was performed as previously described [14]. Briefly, tissues frozen sections were fixed in $1 \%$ formalin in PBS for $1 \mathrm{~min}$ at RT, washed three times in PBS and incubated overnight on X-gal staining solution $[1 \mathrm{mg} / \mathrm{mL}$ of X-gal (VWR), $40 \mathrm{mM}$ citric acid/sodium phosphate buffer, $5 \mathrm{mM}$ potassium ferricyanide (Sigma), $5 \mathrm{mM}$ potassium ferrocyanide (Sigma), $150 \mathrm{mM} \mathrm{NaCl}$, and $2 \mathrm{mM} \mathrm{MgCl} 2$ ] at $37{ }^{\circ} \mathrm{C}$ in a humidified chamber. The experiments were carried out using staining solutions at $\mathrm{pH} 6.0$ to assess the SA- $\beta$-gal activity. Samples were rinsed with distilled water and counterstained with Nuclear Fast Red (Sigma) for 5 min. Images were acquired using Pia-Apochromat $20 \times 0.8 \quad$ M27 objective on Axio scan Z1 (Zeiss).

\section{qRT-PCR}

RNA extraction and qRT-PCR methods were previously described $[29,30]$. Murine primer sequences were listed in Table 3.

\section{Immunoblotting analyses}

Frozen liver tissues were ground under liquid nitrogen and resuspended in RIPA lysis buffer (ThermoFisher) that included protease and phosphatase inhibitors cocktails (Sigma P5726 and P8340). Immunoblotting analyses were performed as previously described [31-33]. Primary Antibodies were purchased from Abcam (CDKN2A/ p16INK4a, ab189034) and Cell Signaling (Histone H3, 9715S). Anti-rabbit IgG HRP-linked (Cell Signaling, 7074 S) was used as secondary antibody. Immuno-positive bands were visualized by chemiluminescence (GE Healthcare Life Sciences) using the Molecular Imager 
Table 3 qRT-PCR primers (Mus Musculus)

\begin{tabular}{lll}
\hline Gene & Forward $\left(5^{\prime}-3^{\prime}\right)$ & Reverse $\left(5^{\prime}-3^{\prime}\right)$ \\
\hline Gapdh & AGGTCGGTGTGAACGGATTTG & TGTAGACCATGTAGTTGAGGTCA \\
p16 & CGCTCTGGCTTTCGTGAACAT & GCCCATCATCATCACCTGGTC \\
Ppar-y & TCGCTGATGCACTGCCTATG & GAGAGGTCCACAGAGCTGATT \\
Cebpa & ACTCCTCCTTTCCTACCG & AGGAAGCAGGAATCCTCC \\
Pgc-1a & TCCCATACACAACCGCAGTC & ACCCTTGGGGTCATTTGGT \\
Plin2 & GACCTTGTGTCCTCCGCTTAT & CAACCGCAATTTGTGGTC \\
Cidec & ATGGACTACGCCATGAAGTCT & CGGTGCTAACACGACAGGG \\
Cd36 & GAGCAACTGGTGGATGGTT & GCAGAATCAAGGGAGAGCAC \\
Timp1 & GCAACTCGGACCTGGTCATAA & CGGCCCGTGATGAGAAACT \\
Col1a1 & GCTCCTCTTAGGGGCCACT & CCACGTCTCACCATTGGGG \\
\hline
\end{tabular}

ChemiDoc xrs + system (Bio-Rad) and quantified by densitometry analysis performed after normalization with H3. Results were expressed as arbitrary units (AU).

\section{Statistical analyses}

Results are expressed as means \pm SD. Comparisons between groups were performed with the parametric Student's t-test, using GraphPad Prism Software (version 5.00 for Windows, San Diego, CA, USA): $p$ value $\leq 0.05$ was considered significant.

\section{Discussion}

The process of aging predisposes to hepatic functional and structural impairment [5]. The most common liver disease, NAFLD can evolve into NASH in the presence of oxidative stress and inflammation [34, 35]. NASH is a serious risk factor for disabling liver diseases such as cirrhosis and HCC. Old age seems to favor NAFLD, $\mathrm{NASH}$, and ultimately $\mathrm{HCC}$, in agreement with the inflamm-aging theory [5]. Others and we have observed an increase in the amount of senescent hepatocytes and other hepatic parenchymal cells during the transition from NAFLD to HCC [32, 36]. In the context of liver disease progression, the concept of selective elimination of senescent cells using senolytics holds a great therapeutic potential [37]. Our present and past work demonstrates that the first generation and best-characterized senolytic cocktail $\mathrm{D}+\mathrm{Q}$, at the same dosage used in literature, is largely ineffective in preventing HCC development and growth in vivo [22]. On the opposite, it is of concern the observation that $\mathrm{D}+\mathrm{Q}$ administration has slight protumorigenic effects in these same models. These data are in marked contrast with the report that treatment with a combination of $D+Q$ reduces NAFLD in HFD-fed mice [18]. However, the latter study did not analyze liver disease progression beyond NAFLD. Recently, Grosse et al. elegantly showed that $\mathrm{D}+\mathrm{Q}$ combined treatment reduced the number of senescent $\mathrm{p} 16^{\text {high }}$ macrophages in the liver and in the adipose tissue, but it had no effect on liver endothelial sinusoidal cells or on adipocytes [38]. In support of our finding, Gross et al. strikingly found that systemic elimination of $\mathrm{p} 16^{\text {high }}$ senescent cells in 10-12 months old mice triggered liver, perivascular tissue fibrosis and general health deterioration [38]. Overall, these controversies suggest that while elimination of some senescent cells is beneficial for healthy aging and overall lifespan [39], it is very important to identify which senescent cells are targeted by specific senolytics and their overall effects on health span.

\section{Conclusions}

In summary, using an animal model that fully recapitulates NAFLD, we demonstrate that $\mathrm{D}+\mathrm{Q}$ treatment is ineffective against age-associated NAFLD-induced HCC. The clinical use of these compounds awaits safety, target identification, and efficacy studies to determine the optimal dosage required to clear senescent cells.

\section{Abbreviations}

NAFLD: Nonalcoholic fatty liver disease; NASH: Nonalcoholic steatohepatitis; HCC: Hepatocellular carcinoma; D: Dasatinib; Q: Quercetin; DEN: Diethylnitrosamine; HFD: High fat diet; SASP: Senescence-associated secretory phenotype; SA- $\beta$-gal: Senescence associated $\beta$-galactosidase; GTT: Glucose tolerance test; H\&E: Hematoxylin \& Eosin; NAS: Histologic activity score; PCR: Polymerase chain reaction; HRP: Horseradish peroxidase; H3: Histone H3; p16: Cyclin Dependent Kinase Inhibitor 2A (CDKN2A/p16INK4a); Gapdh: Glyceraldehyde 3-phosphate dehydrogenase; Ppar-y: Peroxisome proliferator-activated receptor gamma; Cebpa: CCAAT enhancer binding protein alpha; Pgc-1a: Peroxisome proliferator-activated receptor gamma coactivator 1-alpha; Plin2: Perilipin-2; Cidec: Cell death inducing DFFA like effector C; Cd36: Cluster of differentiation 36, also known as fatty acid translocase (FAT); Timp1: Tissue inhibitor of metalloproteinases 1; Col1a1: Collagen type I alpha 1 chain.

\section{Supplementary Information}

The online version contains supplementary material available at https://doi. org/10.1186/s12964-021-00731-0.

\section{Acknowledgements}

The authors thank the members of the Center for Translational Medicine (CTM, ICRC) for support, and Dr. Petra Kovacovicova for help with performing GTT experiments.

\section{Authors' contributions}

MR, KK, JF, OLR and SG carried out all molecular and cellular studies. KK, MF and LL performed the animal experiments. DC performed expert anatomicpathological evaluation on histochemical sections of mice liver biopsies. JAO and AGG participated in the design of the study and performed the statistical analysis. MR, KK, AGG and DC contributed substantially with project supervision, intellectual input and drafting the manuscript. MV originally conceived, designed and coordinated the project, and drafted the manuscript. All authors read and approved the final manuscript.

\section{Funding}

This work was supported by the European Social Fund and European Regional Development Fund_Project MAGNET (No. CZ.02.1.01/0.0/0.0/15_003/00004 92 (MV), by the Ministry of Health of the Czech Republic (No. NV18-03-00058) (MV), by the Ministry of Agriculture of the Czech Republic (project RO0518) (MF), and by the European Commission Horizon 2020 Framework Program (Project 856871—TRANSTEM) (MV). 


\section{Availability of data and materials}

All the data generated or analyzed for this project are included in this article.

\section{Declarations}

\section{Consent for publication}

All authors read and are consent for the publication of the manuscript.

\section{Competing interests}

The authors declare that they have no competing interests.

\begin{abstract}
Author details
1 International Clinical Research Center, St. Anne's University Hospital, Brno, Czech Republic. ${ }^{2}$ Department of Biology, Faculty of Medicine, Masaryk University, Brno, Czech Republic. ${ }^{3}$ Institute for Liver and Digestive Health (ILDH), Division of Medicine, University College London (UCL), London, UK. ${ }^{4}$ Veterinary Research Institute, Brno, Czech Republic. ${ }^{5}$ Department of Health Promotion, Mother and Child Care, Internal Medicine and Medical Specialties, Pathologic Anatomy Unit-University of Palermo, Palermo, Italy. ${ }^{6}$ ERA Chair in Translational Stem Cell Biology, Medical University of Varna, Varna, Bulgaria.
\end{abstract}

\section{Received: 15 December 2020 Accepted: 8 March 2021}

\section{Published online: 08 April 2021}

\section{References}

1. Campisi J. Aging, cellular senescence, and cancer. Annu Rev Physiol. 2013;75:685-705

2. Hodes RJ, Sierra F, Austad SN, Epel E, Neigh GN, Erlandson KM, et al. Disease drivers of aging. Ann NY Acad Sci. 2016;1386(1):45-68.

3. Inouye SK, Studenski S, Tinetti ME, Kuchel GA. Geriatric syndromes: clinical, research, and policy implications of a core geriatric concept. Am Geriatr Soc. 2007;55(5):780-91.

4. Araujo AR, Rosso N, Bedogni G, Tiribelli C, Bellentani S. Global epidemiology of non-alcoholic fatty liver disease/non-alcoholic steatohepatitis: What we need in the future. Liver Int. 2018;38(Suppl 1):47-51.

5. Sheedfar F, Di Biase S, Koonen D, Vinciguerra M. Liver diseases and aging: friends or foes? Aging Cell. 2013;12(6):950-4.

6. McHugh D, Gil J. Senescence and aging: causes, consequences, and therapeutic avenues. J Cell Biol. 2018;217(1):65-77.

7. Childs BG, Gluscevic M, Baker DJ, Laberge RM, Marquess D, Dananberg J, et al. Senescent cells: an emerging target for diseases of ageing. Nat Rev Drug Discov. 2017;16(10):718-35.

8. Schafer MJ, Zhang X, Kumar A, Atkinson EJ, Zhu Y, Jachim S, et al. The senescence-associated secretome as an indicator of age and medical risk. JCI Insight 2020;5(12).

9. Gorgoulis V, Adams PD, Alimonti A, Bennett DC, Bischof O, Bishop C, et al. Cellular Senescence: defining a path forward. Cell. 2019;179(4):813-27.

10. Pignolo RJ, Passos JF, Khosla S, Tchkonia T, Kirkland JL. Reducing senescent cell burden in aging and disease. Trends Mol Med. 2020;26(7):630-8.

11. Kirkland $J$, Tchkonia T. Cellular senescence: a translational perspective. EBioMedicine. 2017;21:21-8.

12. Kirkland JL, Tchkonia T, Zhu Y, Niedernhofer $L$, Robbins PD. The clinica potential of senolytic drugs. J Am Geriatr Soc. 2017;65(10):2297-301.

13. Calimport SRG, Bentley BL, Stewart CE, Pawelec G, Scuteri A, Vinciguerra $\mathrm{M}$, et al. To help aging populations, classify organismal senescence. Science. 2019;366(6465):576-8.

14. Raffaele M, Kovacovicova K, Bonomini F, Rezzani R, Frohlich J, Vinciguerra M. Senescence-like phenotype in post-mitotic cells of mice entering middle age. Aging (Albany NY). 2020;12(14):13979-90.

15. Fuhrmann-Stroissnigg $H$, Santiago FE, Grassi D, Ling Y, Niedernhofer $L$, Robbins PD. SA-beta-Galactosidase-Based Screening Assay for the Identification of Senotherapeutic Drugs. J Vis Exp. 2019(148).

16. Fuhrmann-Stroissnigg H, Ling YY, Zhao J, McGowan SJ, Zhu Y, Brooks RW, et al. Identification of HSP90 inhibitors as a novel class of senolytics. Nat Commun. 2017:8(1):422
17. Zhu Y, Tchkonia T, Pirtskhalava T, Gower AC, Ding H, Giorgadze N, et al. The Achilles' heel of senescent cells: from transcriptome to senolytic drugs. Aging Cell. 2015;14(4):644-58.

18. Ogrodnik M, Miwa S, Tchkonia T, Tiniakos D, Wilson CL, Lahat A, et al. Cellular senescence drives age-dependent hepatic steatosis. Nat Commun. 2017;8:15691

19. Sierra-Ramirez A, Lopez-Aceituno JL, Costa-Machado LF, Plaza A, Barradas M, Fernandez-Marcos PJ. Transient metabolic improvement in obese mice treated with navitoclax or dasatinib/quercetin. Aging (Albany NY). 2020;12(12):11337-48.

20. Ogrodnik M, Zhu Y, Langhi LGP, Tchkonia T, Kruger P, Fielder E, et al. Obesity-induced cellular senescence drives anxiety and impairs neurogenesis. Cell Metab. 2019;29(5):1061-77.

21. Hickson LJ, Langhi Prata LGP, Bobart SA, Evans TK, Giorgadze N, Hashmi SK, et al. Senolytics decrease senescent cells in humans: preliminary report from a clinical trial of Dasatinib plus Quercetin in individuals with diabetic kidney disease. EBioMedicine. 2019;47:446-56

22. Kovacovicova K, Skolnaja M, Heinmaa M, Mistrik M, Pata P, Pata I, et al. Senolytic cocktail Dasatinib+Quercetin $(D+Q)$ does not enhance the efficacy of senescence-inducing chemotherapy in liver cancer. Front Oncol. 2018;8:459

23. Rappa F, Greco A, Podrini C, Cappello F, Foti M, Bourgoin L, et al. Immunopositivity for histone macroH2A1 isoforms marks steatosis-associated hepatocellular carcinoma. PLoS ONE. 2013;8(1):e54458.

24. Park EJ, Lee JH, Yu GY, He G, Ali SR, Holzer RG, et al. Dietary and genetic obesity promote liver inflammation and tumorigenesis by enhancing IL-6 and TNF expression. Cell. 2010;140(2):197-208.

25. Biddinger SB, Almind K, Miyazaki M, Kokkotou E, Ntambi JM, Kahn CR. Effects of diet and genetic background on sterol regulatory elementbinding protein-1C, stearoyl-CoA desaturase 1, and the development of the metabolic syndrome. Diabetes. 2005;54(5):1314-23.

26. Alves-Bezerra M, Cohen DE. Triglyceride metabolism in the liver. Compr Physiol. 2017:8(1):1-8.

27. Cazanave S, Podtelezhnikov A, Jensen K, Seneshaw M, Kumar DP, Min HK, et al. The transcriptomic signature of disease development and progression of nonalcoholic fatty liver disease. Sci Rep. 2017;7(1):17193.

28. Takeda D, Nitta H, Takahara T, Hasegawa Y, Itou N, Wakabayashi G. Effect of preoperative chemotherapy on postoperative liver regeneration following hepatic resection as estimated by liver volume. World J Surg Oncol. 2013;11:65.

29. Raffaele M, Pittala V, Zingales V, Barbagallo I, Salerno L, Li Volti G, et al. Heme oxygenase-1 inhibition sensitizes human prostate cancer cells towards glucose deprivation and metformin-mediated cell death. Int J Mol Sci. 2019;20(10):2593.

30. Raffaele M, Barbagallo I, Licari M, Carota G, Sferrazzo G, Spampinato $\mathrm{M}$, et al. $\mathrm{N}$-acetylcysteine (NAC) ameliorates lipid-related metabolic dysfunction in bone marrow stromal cells-derived adipocytes. Evid Based Complem Alternat Med. 2018;2018:5310961.

31. Frohlich J, Kovacovicova K, Mazza T, Emma MR, Cabibi D, Foti M, et al. GDF11 induces mild hepatic fibrosis independent of metabolic health. Aging (Albany NY). 2020;12(20):20024-46.

32. Borghesan M, Fusilli C, Rappa F, Panebianco C, Rizzo G, Oben JA, et al. DNA hypomethylation and histone variant macroH2A1 synergistically attenuate chemotherapy-induced senescence to promote hepatocellular carcinoma progression. Cancer Res. 2016;76(3):594-606.

33. Benegiamo G, Vinciguerra M, Mazzoccoli G, Piepoli A, Andriulli A, Pazienza V. DNA methyltransferases 1 and 3b expression in Huh-7 cells expressing HCV core protein of different genotypes. Dig Dis Sci. 2012;57(6):1598-603.

34. Shen $\mathrm{HH}$, Alex R, Bellner $\mathrm{L}$, Raffaele M, Licari M, Vanella $\mathrm{L}$, et al. Milk thistle seed cold press oil attenuates markers of the metabolic syndrome in a mouse model of dietary-induced obesity. J Food Biochem. 2020;44(12):e13522

35. Raffaele M, Licari M, Amin S, Alex R, Shen HH, Singh SP, et al. Cold press pomegranate seed oil attenuates dietary-obesity induced hepatic steatosis and fibrosis through antioxidant and mitochondrial pathways in obese mice. Int J Mol Sci. 2020;21(15):5469.

36. Aravinthan AD, Alexander GJM. Senescence in chronic liver disease: Is the future in aging? J Hepatol. 2016;65(4):825-34.

37. Papatheodoridi AM, Chrysavgis L, Koutsilieris M, Chatzigeorgiou A. The role of senescence in the development of nonalcoholic fatty liver 
disease and progression to nonalcoholic steatohepatitis. Hepatology. 2020;71(1):363-74.

38. Grosse L, Wagner N, Emelyanov A, Molina C, Lacas-Gervais S, Wagner KD, et al. Defined p16(high) senescent cell types are indispensable for mouse healthspan. Cell Metab. 2020;32(1):87-99.

39. Xu M, Pirtskhalava T, Farr JN, Weigand BM, Palmer AK, Weivoda MM, et al. Senolytics improve physical function and increase lifespan in old age. Nat Med. 2018;24(8):1246-56.

\section{Publisher's Note}

Springer Nature remains neutral with regard to jurisdictional claims in published maps and institutional affiliations.
Ready to submit your research? Choose BMC and benefit from:

- fast, convenient online submission

- thorough peer review by experienced researchers in your field

- rapid publication on acceptance

- support for research data, including large and complex data types

- gold Open Access which fosters wider collaboration and increased citations

- maximum visibility for your research: over 100M website views per year

At BMC, research is always in progress.

Learn more biomedcentral.com/submissions 